\title{
Context isn't everything: Search performance is influenced by the nature of the task but not the background
}

\author{
Brett A. Cochrane ${ }^{1}$ (D) Jay Pratt ${ }^{1}$ \\ Accepted: 9 November 2020 / Published online: 23 November 2020 \\ (C) The Psychonomic Society, Inc. 2020Abstract
}

It has been demonstrated in the literature that cues in the environment that are predictive of how a task ought to be performed can influence performance. In an extension of this general notion, Cosman and Vecera (Journal of Experimental Psychology: Human Perception and Performance, 39(3), 836-848, 2013) reported that simply performing singleton and feature search tasks when irrelevant scenes were displayed in the background automatically modulated the search strategies adopted by participants when these scenes were reinstated at a later time. While intriguing, this result was also somewhat surprising given that an adaptive system (like the human brain) should disregard irrelevant information so task competencies generalize across environments. To investigate this issue further, we replicated the experimental procedures of Cosman and Vecera, while varying whether the test phase was either a singleton search (Experiments 1 and 3) or a feature search (Experiment 2) task. While it was observed that the nature of the search task varied whether a color singleton distractor influenced performance, there was no evidence that background scenes modulated the search strategies adopted by participants, contrasting the results of Cosman and Vecera. Overall, the findings here support the conclusion that the visual system prioritizes task-relevant information while disregarding irrelevant background information.

Keywords Visual search $\cdot$ Attentional capture $\cdot$ Scene Perception

\section{Introduction}

The visual system is constantly integrating information from the surrounding environment to inform us how we ought to perform a task. At the same time, we need to be able to separate background context information from the task being performed so that task competencies can generalize to new environments. This dilemma highlights that the visual system needs to perform a balancing act between utilizing context information when it is relevant to a task and disregarding it when it is not.

Perhaps not surprisingly, one factor that our visual system utilizes to inform how a task ought to be performed is the nature of the task itself. This notion is highlighted by the seminal work of Bacon and Egeth (1994) demonstrating that attention capture of a color singleton can be attenuated by the type of search task performed. Participants in their study

Brett A. Cochrane

brett.cochrane@utoronto.ca

1 Department of Psychology, University of Toronto, Sidney Smith Hall, 100 St. George Street, Toronto, Ontario M5S 3G3, Canada performed either a singleton search task where they had to find an oddball shape target amongst homogeneous distractor shapes or a feature search task where they had to find a circle target amongst a mostly heterogeneous set of shape distractors. The researchers observed that when participants performed the singleton search task, responses were slower when the color singleton distractor was present in the search display than if it was absent. However, the color singleton distractor had no impact when participants performed the feature search task, suggesting that the nature of the search task changed how search was performed. In other words, if it was the goal to find the oddball shape, attention was captured by other types of oddballs (i.e., color oddballs), whereas if the task was to find a specific feature (i.e., a circle), oddballs no longer captured attention.

In an important extension to the findings of Bacon and Egeth (1994), Leber and Egeth (2006b) explored whether prior search experience would change whether participants adopted a singleton or a feature search strategy. Leber and Egeth had participants perform both a training and test phase. In the training phase, participants either performed a singleton search task where they had to find the oddball target object amongst homogeneous distractor objects, or a feature search task where they had to find the circle target amongst a set of 
mostly heterogeneous distractor objects. Following the training phase, participants performed a test phase where they had to find a circle target amongst homogeneous diamond distractors. Leber and Egeth observed that there was a greater influence of the color singleton distractor on participants that trained the singleton search task than those that trained the feature search task, suggesting that prior experience can affect the search strategy adopted by participants.

While there is intuitive appeal to the notion that past search can influence how future search tasks are performed (Leber \& Egeth, 2006b; see also Leber, Kawahara, \& Gabari, 2009), an interesting variant to this line of research was reported by Cosman and Vecera (2013) when they examined the effect of task-irrelevant contexts. Cosman and Vecera used an experimental procedure similar to Leber and Egeth, except that participants trained both singleton and feature search across blocks of trials in the training phase. Importantly, participants always performed the singleton search task with one type of background scene (e.g., a city scene) and the feature search task with a different background scene (e.g., a forest scene). During the test phase, participants had to find a circle amongst homogeneous diamond distractors while the singleton and feature search background scenes varied randomly on a trial-by-trial basis. Even though the background scenes were irrelevant to the task, search performance was significantly impaired by the singleton color distractor when the background scenes associated with singleton search were displayed. When the background scenes associated with feature search were displayed, now the singleton distractor had no influence on search performance. In other words, these irrelevant background scenes were impactful enough to automatically modulate the search strategy adopted by participants on a trial-by-trial basis.

The finding that irrelevant background information impacted a visual search task by Cosman and Vecera (2013) is intriguing and somewhat surprising. From a conceptual point of view, if you were designing an intelligent system to play chess, you would want a system that can separate relevant information critical to winning the game from all other irrelevant information (like the texture of the game board). From an empirical point of view, while there is good evidence that context effects can be quite robust (Chun \& Jiang, 1998; Crump, Gong, \& Milliken, 2006; Jacoby, Lindsay, \& Hessels, 2003; Leber, Gwinn, Hong, \& O'Toole, 2016; Wang \& Theeuwes, 2018), in these studies context was inextricably linked to how the task ought to be performed (i.e., the context was task relevant). Further, there are studies demonstrating that background context does not influence performance even when it is informative (Allon \& Leber, 2019; Britton \& Anderson, 2020; Brooks, Rasmussen, \& Hollingworth, 2010). In Cosman and Vecera, however, not only did the irrelevant background scenes have a profound impact on performance, the irrelevant context essentially eliminated the contribution from the (relevant) task itself. That is, the presence of a singleton color distractor in singleton search tasks like that used by Cosman and Vecera have consistently been shown to slow responding relative to if they were absent (Bacon \& Egeth, 1994; Gaspelin \& Luck, 2018; Pashler, 1988; Theeuwes, 1992), but this was not the case in Cosman and Vecera. Rather, they found a situation in which the irrelevant background information had a greater impact on search performance than the nature of the task itself.

Given the unexpected nature of Cosman and Vecera's (2013) findings, and the scope of the consequences of these findings, we sought to re-examine the influence of background scenes on visual search. To do so, we replicated and extended the experimental procedures of Cosman and Vecera while varying the nature of the visual search task of the test phase across experiments. More specifically, Experiment 1 constituted a close replication of Cosman and Vecera; Experiment 2 assessed whether these context effects extended to feature search; and Experiment 3 assessed whether the integration of context and search task was important. In all cases there was a training phase where participants performed singleton and features search tasks with different background scenes (i.e., a city or forest) across blocks of trials. In the following test phase, participants either performed a singleton search task where they had to find a circle target amongst homogeneous distractor diamonds (Experiments 1 and 3) or a feature search task where they had to find a circle target amongst a mostly heterogeneous set of distractor shapes (Experiment 2). Critically, the singleton and feature search background scenes of the training phase were randomly displayed on a trial-by-trial basis in the test phase, and a singleton color distractor was present on some trials and absent on others. If the background scenes influence how the visual search task was performed, the presence of the color singleton distractor should slow responding when the singleton search backgrounds were displayed compared to the feature search backgrounds. If the background scenes do not influence the visual search task, we should observe similar effects of the color singleton distractor across background scenes.

\section{Experiment 1}

The purpose of Experiment 1 was to closely replicate Cosman and Vecera (2013). Across the experimental session, participants had to find the target and indicate whether the line on the inside of it was tilted to either the right or the left. Participants performed this task during the training and test phases of the experiment. During the training phase, participants performed alternating blocks of singleton and feature search. In the singleton search task, the target was defined as the oddball shape amongst homogeneous distractor shapes. In the feature search task, the target was always a circle amongst a diamond, triangle, square, and two other shapes (diamond, triangle, or square) that were randomized on a trial-by-trial basis. In the 


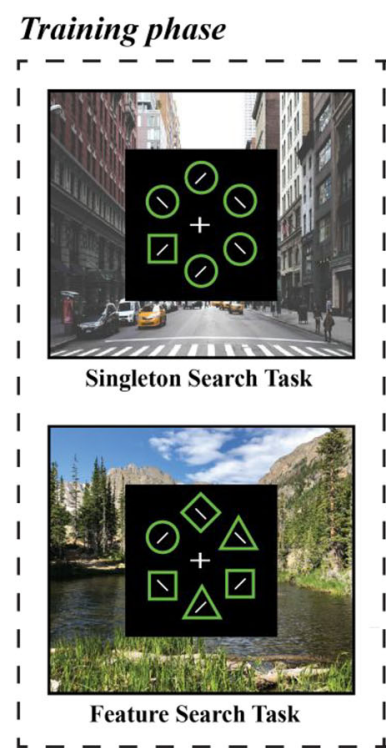

Fig. 1 The left panel depicts an example of the training search displays for one of the counterbalancing schemes of Experiments 1 and 2. The right panel depicts an example of a color singleton distractor present trial

following test phase, the target was always a circle amongst homogeneous diamond distractors and the singleton and feature backgrounds were displayed randomly on a trial-by-trial basis. On half of the trials of the training and test phases of the experiment, a singleton color distractor was presented and on the other half of trials it was absent. If Cosman and Vecera's results are observable upon replication, the color singleton distractor should slow performance when the background of the singleton search task is displayed and have no influence when the background of the feature search task is displayed. ${ }^{1}$

\section{Methods}

Participants Eighteen undergraduates at the University of Toronto participated in exchange for course credit or $\$ 10$ monetary compensation (10 females, $M_{\text {age }}=19.7$ years). All participants had normal color vision and normal or correctedto-normal vision. A sample size of 18 participants was selected so that we would have equivalent power relative to Cosman and Vecera $(2013 ; \mathrm{n}=17)$ while also fully counterbalancing key conditions.

Apparatus and stimuli Stimuli were presented using PsychoPy v3.1.5 on an LED monitor with a $144-\mathrm{Hz}$ refresh

\footnotetext{
${ }^{1}$ While the present experiment is a close replication of Cosman and Vecera (2013)'s first experiment, it is not identical. There are four key differences: (1) We fully counterbalance the assignment of forest and city background scenes with the singleton and feature search tasks. (2) The distractors in the singleton search task could be a homogeneous set of circles, squares, diamonds, or triangles - they were always diamonds in Cosman and Vecera's study (although see Experiment 3). (3) Our stimuli were slightly smaller than Cosman and Vecera's stimuli. (4) we had participants report the background scenes on a trial-by-trial basis during the test phase of the experiment
}

when the singleton search context was reinstated. In the test phase, participants performed a singleton search task in Experiment 1 and a feature search task in Experiment 2

rate. A white fixation cross was presented centrally that subtended a horizontal and vertical visual angle of $.3^{\circ}$ and had a luminance value of $73.08 \mathrm{~cd} / \mathrm{m}^{2}$. All search displays consisted of six outline shapes that were equally spaced around an invisible circle that had a radius of $3.0^{\circ}$ of visual angle. There were four outlined shapes that were used throughout the experiment: a circle, square, triangle, and diamond. The sides of the square and diamond subtended $1.0^{\circ}$ of visual angle, the circle had a diameter of $1.25^{\circ}$ of visual angle, and the sides of the triangle subtended $1.46^{\circ}$ of visual angle. The outlined shapes were presented in green that had a luminance value of $23.63 \mathrm{~cd} / \mathrm{m}^{2}$, and the singleton distractor was presented in red that had a luminance value of $23.56 \mathrm{~cd} / \mathrm{m}^{2}$. Each shape contained a white line that subtended $.75^{\circ}$ of visual angle and had a luminance value of $73.08 \mathrm{~cd} / \mathrm{m}^{2}$. Forest and city scenes subtended $16.67^{\circ}$ of horizontal and $12.5^{\circ}$ of vertical visual angle. Search displays were presented on a black background in front of the scene displays and subtended $8.0^{\circ}$ vertical and horizontal visual angle.

Procedure and design Participants' placed their head on a chin and head rest that was positioned $57 \mathrm{~cm}$ from the monitor. Each trial began with the presentation of the background scene and fixation cross on a black background for 1,000 ms. Following this, the search display was presented on-screen until participants made a response. Participants were instructed to locate the target and indicate whether the line inside the target was tilted either $45^{\circ}$ to the left or right. They made a left response by pressing the " $Z$ " key and a right response by pressing the "M" key on a standard QWERTY keyboard. On half of the trials, one of the distractors was a color singleton and on the other half of trials, the color singleton was absent from the search display. This was the basic 
procedure throughout the experimental session. An example of the experimental procedure is depicted in Fig. 1.

The experimental session was split into training and test phases. In the training phase, participants performed two types of search that were separated by block: singleton and feature search. In the singleton search task, the target was defined as the oddball shape that differed from the homogeneous distractor shapes (e.g., a triangle amongst circles). In the feature search task, the target was always a circle amongst a square, diamond, triangle, and two other distractor shapes that were randomized on a trial-by-trial basis. Each type of search was paired with either the city or forest scenes across blocks. The scene and search type pairings were fully counterbalanced across participants. Participants performed four blocks of 36 trials of each of the singleton and feature search tasks -144 trials for each search task (288 total). Following each block, participants had to report whether the background scene was a city or a forest by keypress. The singleton and feature blocks alternated across the training phase, and the type of search task in the first block was randomized for each participant.

Once participants completed the training phase, they performed the test phase of the experiment. Here, the target was always a circle amongst homogeneous diamond distractor shapes. Background scenes now changed on a trial-by-trial basis and there were equal proportions of singleton- and feature-search background scenes for the singleton color distractor-present and absent conditions. Following each trial, participants reported whether the background was a city or a forest - this design feature was implemented given that it has been proposed that attention to background scenes can strengthen context-based search effects (Allon \& Leber, 2019). Participants completed three blocks of 72 trials in the test phase of the experiment (216 total trials).

\section{Results}

Training phase Response times (RTs) that were less than $200 \mathrm{~ms}$ and exceeded 1,500 ms were excluded from analyses, which excluded $25.8 \%$ of observations. ${ }^{2}$ The remaining correct RTs and error rates were submitted to within-subject ANOVAs that treated search task (feature/singleton) and singleton distractor presence (present/absent) as factors. For all analyses in this study, an alpha criterion of .05 was used to determine statistical significance. Mean RTs for the training phase are depicted in Table 1.

\footnotetext{
${ }^{2}$ All analyses of Experiments 1 and 2 were also conducted with an outlier elimination procedure that excluded observations with RTs less than $200 \mathrm{~ms}$ and greater than $3,000 \mathrm{~ms}$ in the training phase, and observations with RTs less than $200 \mathrm{~ms}$ and greater than $2000 \mathrm{~ms}$ in the test phase. These removal procedures eliminated $2.7-9.6 \%$ of observations across the testing and training phases of Experiments 1 and 2. This relative liberal outlier removal procedure did not have a meaningful impact on the results of the study - that is, any result that was significant, remained significant and any result that was not significant, remained not significant.
}

The RT analysis revealed that there was a significant main effect of singleton distractor presence, $F(1,17)=12.8, p=$ $.002, \eta_{\mathrm{p}}^{2}=.43$, reflecting slower performance when the singleton distractor was present than absent. There was also a significant main effect of search task, $F(1,17)=167.5, p<$ $.001, \eta_{\mathrm{p}}^{2}=.91$, reflecting slower performance in the singleton search task than in the feature search task. These main effects were qualified by a significant interaction of singleton distractor presence and search task, $F(1,17)=5.17, p=.04$, $\eta_{\mathrm{p}}^{2}=.23$. This interaction was explored further by evaluating the effect of singleton distractor presence for the singleton and feature search tasks separately. The analysis of the feature search task did not reveal a significant effect of singleton distractor presence $(p=.44)$, reflecting similar RTs when the singleton distractor was present $(765 \mathrm{~ms})$ and absent $(757 \mathrm{~ms})$. The analysis of the singleton search task revealed a significant effect of distractor presence, $t(17)=3.74, p=.002, d=.34$, reflecting slower responses when the singleton distractor was present $(1,081 \mathrm{~ms})$ than absent $(1,032 \mathrm{~ms})$.

No effect in the analysis of error rates neared significance (all $F<1$ ) with the exception of the interaction of singleton distractor presence and search type $(p=.09)$. See Table 1 for mean percentage error rates.

Test phase RTs that were less than 200 ms or greater than $1,500 \mathrm{~ms}$ were removed from analyses, which led to the removal of $19.0 \%$ of observations. The remaining correct RTs and error rates from the test phase (see Fig. 2) were submitted to within-subject ANOVAs that treated context (singleton/feature) and singleton distractor presence (present/absent) as factors.

The analysis of RTs revealed a significant main effect of singleton distractor presence, $F(1,17)=15.1, p<.001, \eta^{2}=$ .47 . Both the main effect of context $(p=.31)$, and the interaction of singleton distractor presence and context $(p=.88)$ were

Table 1 Mean response times (RTs; ms) and error percentages (\%E) for the feature and singleton search tasks of the training phase of Experiments 1,2 , and 3

\begin{tabular}{rcccc}
\hline & \multicolumn{3}{l}{ Singleton distractor RT } & \\
\cline { 2 - 5 } & Present & & Absent & \\
\hline Experiment 1 & Mean & $\% \mathrm{E}$ & Mean & $\% \mathrm{E}$ \\
Feature Search & 765 & 5.8 & 757 & 6.8 \\
Singleton Search & 1,082 & 6.1 & 1,032 & 4.7 \\
Experiment 2 & & & & \\
Feature Search & 699 & 2.6 & 680 & 2.8 \\
Singleton Search & 1,016 & 4.5 & 972 & 3.1 \\
Experiment 3 & & & & \\
Feature Search & 918 & 4.7 & 908 & 4.3 \\
Singleton Search & 1,394 & 8.7 & 1,333 & 7.3 \\
\hline
\end{tabular}


not significant. The main effect of distractor presence reflected faster responses when the singleton color was absent $(882 \mathrm{~ms})$ than present $(910 \mathrm{~ms})$ in the feature search context, and when it was absent $(875 \mathrm{~ms})$ than present $(900 \mathrm{~ms})$ in the singleton search context.

The analysis of error rates revealed no significant effects (all $F<2$ ). Error rates are depicted in Table 2.

Probe analysis We conducted additional analyses to evaluate whether the present results were influenced by the trial-bytrial question of whether the background scene was a forest or a city. First, participants incorrectly answered this question $4.6 \%$ of the time and these error rates did not differ across scenes $(p=.20)$. Second, none of the RT analyses of the test phase changed in a meaningful way when excluding participants that failed to accurately report the background - that is, the significant results remained significant and the not significant results remained not significant.

Block analysis A possible reason why the above analyses failed to demonstrate an effect of context was that the association between context and the search task degraded across trials of the test phase. To evaluate this issue, we separated trials into six blocks based on the order in which they were performed, and submitted the corresponding RTs to separate within-subject ANOVAs that treated context (feature/singleton) and singleton distractor presence (present/absent) as factors. These analyses revealed that the interactions of search task and singleton distractor presence was never close to significant (all $F<2)$ with exception of the third block $(p=.06)$. It is noteworthy that this near significant effect was due to a larger singleton capture effect in the feature context relative to the singleton context - a pattern of results in the opposite direction of that predicted by Cosman and Vecera (2013).

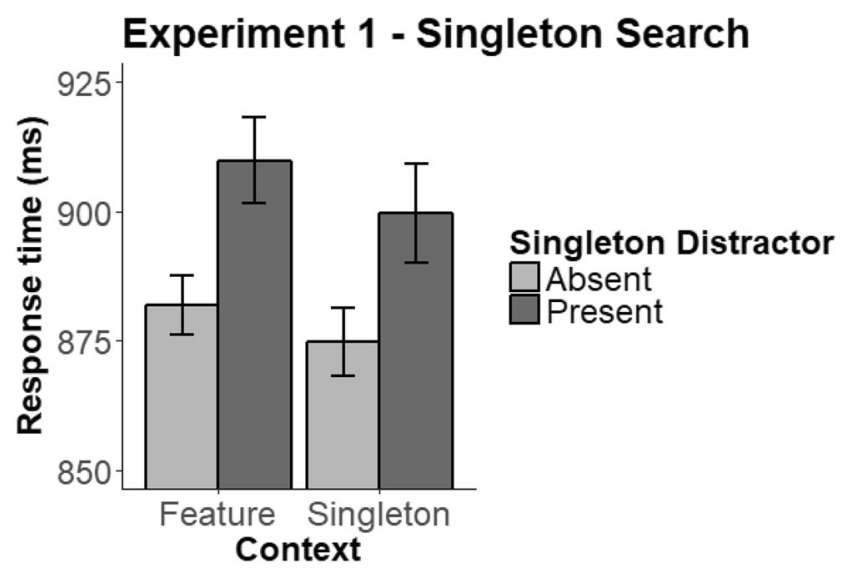

Fig. 2 Mean response times when the feature and singleton contexts were reinstated in the test phase of Experiment 1. Error bars represent the standard error of the mean corrected to remove between-subject variability (Cousineau, 2005; Morey, 2008)
Table 2 Mean error percentages $(\% \mathrm{E})$ for the feature and singleton contexts of the singleton distractor conditions across Experiments 1, 2, and 3

\begin{tabular}{rcc}
\hline & \multicolumn{2}{c}{ Singleton distractor \% } \\
\cline { 2 - 3 } & Present & Absent \\
\hline $\begin{array}{r}\text { Experiment 1 } \\
\text { Feature Context }\end{array}$ & 2.6 & 1.9 \\
Singleton Context & 1.4 & \\
Experiment 2 & 1.3 & 2.3 \\
Feature Context & 0.7 & 1.0 \\
Singleton Context & 3.5 & 0.8 \\
Experiment 3 & 3.3 & 3.1 \\
Feature Context & & 3.2 \\
Singleton Context & & \\
\hline
\end{tabular}

\section{Discussion}

Unlike Cosman and Vecera (2013), we did not find any evidence that background context influenced the search strategy adopted by participants, despite using very similar methods. Instead, the nature of the search task appeared to be the sole determinant of whether the singleton color distractor influenced performance. That is, when participants performed the singleton search task in either the test or training phases of the experiment, the presence of a color singleton distractor slowed responding relative to when it was absent. In the feature search task of the training phase, however, responses were unaffected by the presence of the color singleton distractor.

\section{Experiment 2}

Experiment 1 demonstrated that only the nature of the search task determined whether the singleton color distractor influenced performance. If this finding is generalizable, we should observe that if the singleton search task of the test phase is changed to a feature search task, the color singleton distractor should no longer influence performance. Further, to the main purpose of this study, if the background scenes influence the search strategy used by participants as espoused by Cosman and Vecera (2013), it would imply that these backgrounds should influence performance irrespective of the type of search task. That is, if the singleton search backgrounds of the training phase cause participants to enter a singleton search mode when reinstated in the test phase, attention should be guided to the singleton color distractor even during a feature search task. Accordingly, Experiment 2 will act as a conceptual replication of both the previous experiment and Cosman and Vecera to further evaluate whether it is the nature of the search task or background scenes that primarily influence search task performance. 


\section{Methods}

Participants Eighteen undergraduates at the University of Toronto participated in exchange for course credit or $\$ 10$ monetary compensation ( 15 females, $M_{\text {age }}=19.2$ years). All participants had normal color vision and normal or correctedto-normal vision. A sample size of 18 participants were selected based on the sample size of Experiment 1.

Apparatus and stimuli Apparatus and stimuli were identical to Experiment 1.

Procedure and design The procedure was identical to Experiment 1 with the exception that in the test phase participants performed a feature search task. Specifically, during the test phase the target was always a circle amongst a square, diamond, triangle, and two other shapes that were randomized to be either a square, diamond, or triangle.

\section{Results}

Training phase RTs less than $200 \mathrm{~ms}$ and greater than 1,500 ms were removed from analyses, which led to the removal of $16.0 \%$ of observations. The remaining correct RTs and error rates from the training phase (see Table 1) were submitted to within-subject ANOVAs that treated search task (singleton/feature) and singleton distractor presence (present/absent) as factors.

The analysis of RTs revealed a significant main effect of singleton distractor presence, $F(1,17)=12.0, p=.002, \eta_{\mathrm{p}}^{2}=$ .41 , reflecting that responses were slower when the singleton distractor was present than absent. There was also a significant main effect of search task, $F(1,17)=270.3, p<.001, \eta^{2}=.94$, reflecting that responses were faster in the feature search task than in the singleton search task. The main effects of singleton distractor presence and search task were qualified by a significant interaction, $F(1,17)=5.78, p=.03, \eta_{p}^{2}=.25$. This interaction was investigated further by conducting additional analyses that evaluated the effect of singleton distractor presence for the singleton and feature search tasks separately. The analysis of the feature search task revealed a significant effect of singleton distractor presence, $t(17)=2.32, p=.03, d=.19$, reflecting faster responses when the singleton distractor was absent $(680 \mathrm{~ms})$ than present $(699 \mathrm{~ms})$. The analysis of the singleton search task revealed a significant effect of singleton distractor presence, $t(17)=3.55, p=.002, d=.30$, reflecting faster responses when the singleton distractor was absent $(972$ $\mathrm{ms})$ than present $(1,016 \mathrm{~ms}){ }^{3}$ The significant interaction of singleton distractor presence and search task therefore constituted a larger singleton distractor effect in the singleton search task than in the feature search task.

\footnotetext{
$\overline{3}$ All Cohen's $d$ values reported in this manuscript were based on aggregate measures of performance in each condition for each participant.
}

The analysis of error rates in the training phase revealed a significant effect of search task, $F(1,17)=5.76, p=.03, \eta^{2}{ }_{p}=$ .25 , reflecting higher error rates for the singleton search task than the feature search task. All other effects in the analysis of error rates were not significant (all $F<2.5$ ). Mean error percentages are presented in Table 1.

Test phase RTs less than $200 \mathrm{~ms}$ and greater than 1,500 ms were removed from analysis, which led to the removal of $11.2 \%$ of observations. The remaining mean correct RTs and error rates from the test phase (see Fig. 3) were submitted to within-subject ANOVAs that treated context (singleton/feature) and singleton distractor presence (present/absent) as factors.

The analysis of RTs revealed no significant main effects of context $(p=1)$, and singleton distractor presence $(p=.19)$. Once again, the interaction of context and singleton distractor presence was not significant $(p=.68)$. This analysis reflected that RTs were similar for the singleton color distractor-present (887 ms) and -absent ( $893 \mathrm{~ms}$ ) conditions in the feature context, and for the singleton color distractor-present (890 ms) and -absent (891 ms) conditions in the singleton context.

There were no significant effects in the analysis of error rates (all $F<1.5$ ) - see Table 2 for the error rates of Experiment 2.

Probe analysis We conducted additional analyses to evaluate whether the results of the test phase were influenced by the trial-by-trial question of whether the background scene was a forest or a city. First, participants incorrectly answered this question $4.0 \%$ of the time and these error rates did not differ across scenes $(p=.90)$. Second, none of these RT analyses changed in a meaningful manner when excluding participants that failed to accurately report the background - once again, all significant results remained significant and the nonsignificant results remained non-significant.

Block analysis Once again, we separated trials into six blocks based on the order in which they were performed, and submitted the corresponding RTs to separate within-subject ANOVAs that treated context (feature/singleton) and singleton distractor presence (present/absent) as factors. These analyses revealed that the interactions of search task and singleton distractor presence were not significant in any of the six blocks (all $F<2$ ), with the exception of block $6, F(1,17)=$ 4.66, $p=.05, \eta_{\mathrm{p}}^{2}=.22$, which indicated a pattern of results opposite to that predicted by Cosman and Vecera (2013).

\section{Comparison of Experiments 1 and 2}

We conducted an additional analysis that evaluated whether the different search tasks in the test phase of Experiments 1 and 2 produced different patterns of results. This analysis was a mixed-factor ANOVA that treated context (singleton/ 


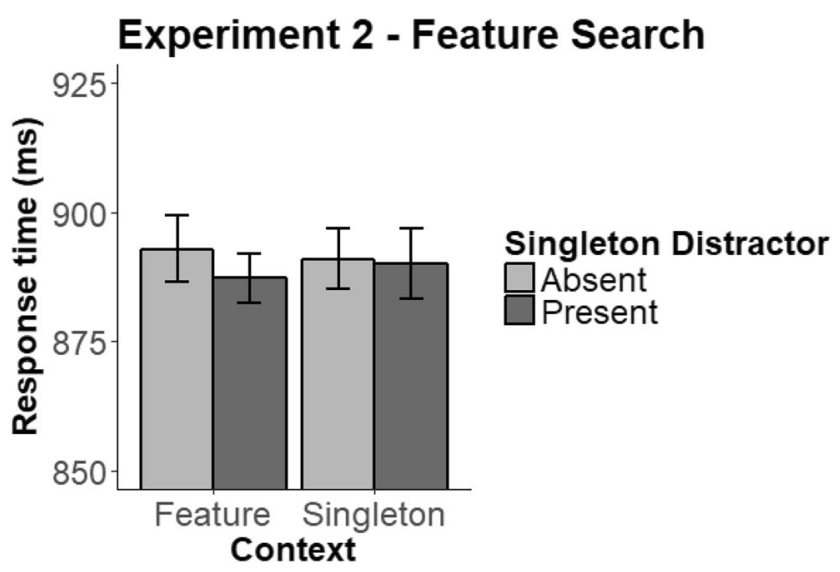

Fig. 3 Mean response times when the feature and singleton contexts were reinstated in the test phase of Experiment 2. Error bars represent the standard error of the mean corrected to remove between-subject variability (Cousineau, 2005; Morey, 2008)

feature) and singleton distractor presence (present/absent) as within-subject factors and experiment $(1 / 2)$ as a betweensubjects factor. This analysis revealed a significant main effect of singleton distractor presence, $F(1,34)=5.62, p=.02, \eta_{\mathrm{p}}^{2}=$ .14. Importantly, this analysis revealed a significant interaction of singleton distractor presence and experiment, $F(1,34)=$ $9.27, p=.004, \eta_{\mathrm{p}}^{2}=.21$, reflecting that the effect of singleton distractor presence was attenuated in the feature search task of Experiment 2 relative to the singleton search task of Experiment 1.

\section{Discussion}

The purpose of Experiment 2 was to further evaluate whether the nature of the search task determined whether a color singleton distractor impacted performance. First, we observed the same results upon replication of the training phase of Experiment 1, finding that a color singleton distractor significantly slowed performance in the singleton search task relative to the feature search task. Second, we observed that when the test phase was changed from a singleton search task to a feature search task the singleton distractor no longer influenced performance, finding once again that the nature of the search task was critical to how search was performed. This finding was further supported by an additional analysis of the test phase of Experiments 1 and 2, which revealed that the presence of a singleton color distractor did indeed slow performance in the singleton search task of Experiment 1 relative to the feature search task of Experiment 2. Third and most important, we once again did not find any evidence that the singleton and feature search contexts could influence performance, contrasting the results of Cosman and Vecera (2013).

\section{Experiment 3}

So far we have demonstrated that reinstating the background scenes associated with singleton and feature search did not influence how search was performed when closely replicating the procedure of Cosman and Vecera (2013) in Experiment 1 and then conceptually replicating their procedure in Experiment 2. Although both experiments converged on the same result, a possible limitation, which was present in the Cosman and Vecera study as well, is that context was constituted as a border that surrounded an otherwise typical search display. It may be that these borders do not produce a sufficient association between background and search task. To put another way, it may be that background information needs to be integrated with the search array in order to have an influence. To evaluate this possibility, we replicated the experimental procedures of Experiment 1 with the exception that the search array now directly overlaid the background display. If an effect of context depends on its integration with the search array, the singleton distractor should have a weaker influence when the feature search background was reinstated relative to the singleton search background. If, once again, the influence of the singleton distractor is determined by the nature of the search task, its presence should slow responding equally across singleton and feature search backgrounds. We also substantially increased the sample size $(n=60)$ in order to ensure that if there was an effect of background, we would have the power to detect it.

Participants Sixty-seven participants were recruited through Prolific (prolific.co) and participated in exchange for $\$ 5$ monetary compensation (29 females, $\mathrm{M}_{\text {age }}=27.6$ years). All participants reported having normal color vision and normal or corrected-to-normal vision. Any participant with below 65\% accuracy across all experimental trials of the test phase was replaced by a new participant until the data for 60 viable participants was collected - this led to the removal of seven participants. The decision to have a sample size of 60 was made a priori - we reasoned that having more than three times the power as that of Cosman and Vecera (2013) should be sufficient to observe their effects provided they were indeed observable.

Apparatus and stimuli The experiment was designed using PsychoPy v2020.2.3 and presented with Pavlovia (pavlovia. org). The experiment was performed using the participants' web browser, computer, and monitor. Stimulus dimensions were sized based on a $1 \times 1$ grid that was centered in the web browser window - this method was used to ensure that the 
relative search array dimensions were consistent across participants while also ensuring that the stimuli would be universally visible for different monitor specifications. The stimuli were squares, circles, diamonds, and triangles. The sides of the square and the diamond subtended a .10 grid proportion, the circle subtended a diameter of 11 grid proportion, and the sides of the triangle subtended a .12 grid proportion. All search displays consisted of six outline shapes that were equally spaced around an invisible circle that had a radius of .5 grid proportion. The shapes were presented in green and the singleton distractor was presented in red. The forest and city scenes subtended .75 vertical and 1 horizontal grid proportions.

Procedure and design The procedure and design was identical to Experiment 1 with the following exceptions: we no longer stabilized head position and ensured participants were equidistant from the monitor. The distractors in the singleton training phase were now always diamonds. ${ }^{4}$ Participants now performed five trials of the singleton search task and five trials of the feature search task prior to the experimental trials. The practice trials were performed without the forest and city backgrounds and participants were provided feedback on their accuracy following each trial. Participants no longer reported the city and forest scenes on a trial-by-trial basis. The search arrays were now overlaid directly onto the forest and city background scenes.

\section{Results}

Training phase RTs less than $200 \mathrm{~ms}$ and greater than $3,000 \mathrm{~ms}$ were excluded from analyses, which excluded $5.6 \%$ of observations. ${ }^{5}$ The remaining correct RTs and error rates were submitted to within-subject ANOVAs that treated search task (feature/singleton) and singleton distractor presence (present/absent) as factors. Mean RTs for the training phase are depicted in Table 1.

The RT analysis revealed that there was a significant main effect of singleton distractor presence, $F(1,59)=16.4, p<$ $.001, \eta_{\mathrm{p}}^{2}=.22$, reflecting slower responses when the singleton distractor was present than absent. There was also a significant main effect of search task, $F(1,59)=319.4, p<.001, \eta_{\mathrm{p}}^{2}=.84$, reflecting slower responses in the singleton search task than in feature search task. There was a significant interaction of singleton distractor presence and search task, $F(1,59)=8.55, p=$ $.005, \eta_{\mathrm{p}}^{2}=.13$. This interaction was explored further by evaluating the effect of singleton distractor presence for the

\footnotetext{
4 The decision to always use diamonds as distractors in the singleton search training phase was made so this experiment was similar to Cosman and Vecera (2013).

${ }^{5}$ We made the a priori decision to use an outlier elimination procedure that included more observations relative to Experiments 1 and 2 across the training and test phases of Experiment 3.
}

singleton and feature search tasks separately. The analysis of the feature search task did not reveal a significant effect of singleton distractor presence $(p=.15)$, reflecting similar RTs when the singleton distractor was present $(918 \mathrm{~ms})$ and absent (908 ms). The analysis of the singleton search task revealed a significant effect of distractor presence, $t(59)=3.83, p<.001$, $d=.22$, reflecting slower responses when the singleton distractor was present $(1,394 \mathrm{~ms})$ than absent $(1,333 \mathrm{~ms})$.

The analysis of error rates revealed a significant effect of search task, $\mathrm{F}(1,59)=9.86, p=.003, \eta_{\mathrm{p}}^{2}=.14$, reflecting higher error percentages in the singleton search task relative to the feature search task. No other effects in the analysis of error rates were significant (all $F<2$ ). See Table 1 for mean percentage error rates.

Test phase RTs that were less than $200 \mathrm{~ms}$ or greater than $2,000 \mathrm{~ms}$ were removed from analysis, which led to the removal of $1.8 \%$ of observations. The mean RTs and error rates from the test phase (see Fig. 4) were submitted to withinsubject ANOVAs that treated context (singleton/feature) and singleton distractor presence (present/absent) as factors.

The analysis of RTs revealed a significant main effect of singleton distractor presence, $F(1,59)=4.48, p=.04, \eta_{\mathrm{p}}^{2}=$ .07 . The main effect of context was not significant $(p=.67)$, and importantly, the interaction of singleton distractor presence and context was also not significant $(p=.62)$. The main effect of distractor presence reflected faster responses when the singleton color was absent $(750 \mathrm{~ms})$ than present $(757 \mathrm{~ms})$ in the feature search context than when it was absent $(747 \mathrm{~ms})$ than present $(757 \mathrm{~ms})$ in the singleton search context.

There were no significant effects in the analysis of error rates (all $F<.5$ ). The mean error percentages for Experiment 3 are depicted in Table 2.

Block analysis As in previous experiments, we separated trials into six blocks based on the order in which they were

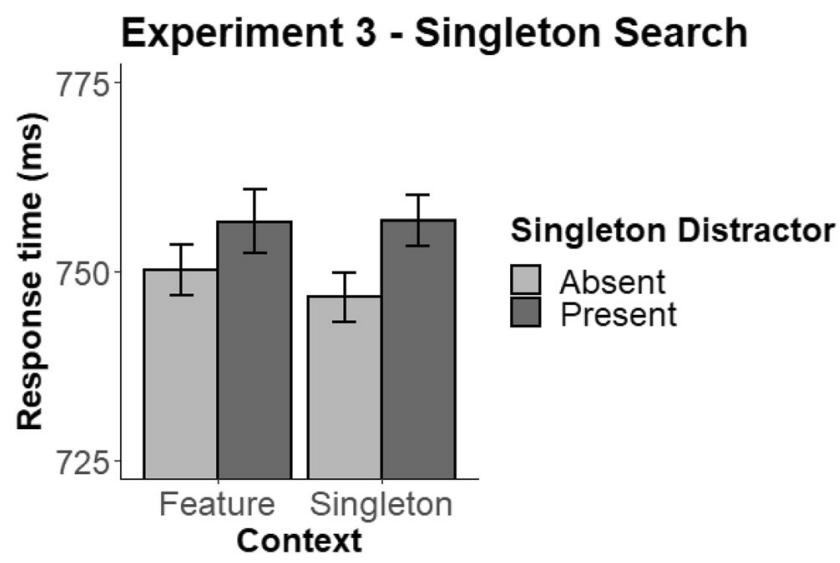

Fig. 4 Mean response times when the feature and singleton contexts were reinstated in the test phase of Experiment 3. Error bars represent the standard error of the mean corrected to remove between-subject variability (Cousineau, 2005; Morey, 2008) 
performed, and submitted the corresponding RTs to separate within-subject ANOVAs that treated context (feature/singleton) and singleton distractor presence (present/absent) as factors. These analyses revealed that the interactions of context and singleton distractor presence were not significant in any block (all $F<2$ ) with exception of the fifth block, $F(1,58)=$ $5.00, p=.03, \eta_{\mathrm{p}}^{2}=.08$, which revealed a pattern of results in keeping with that espoused by Cosman and Vecera (2013). ${ }^{6}$

\section{Discussion}

The purpose of Experiment 3 was to assess whether the contexts associated with singleton and feature search tasks could influence performance when they were integrated with the search arrays. It was revealed that even when these singleton and feature search contexts were integrated they still did not influence performance. Instead, the color singleton distractor significantly slowed responding in both the singleton search task of the training and test phases and was absent in the feature search task, supporting the notion that the nature of the search task can have a profound impact on search performance and background information does not. It is noteworthy that while the effects of the color singleton distractor were present, they were attenuated relative to Experiment 1 . We suspect that the integration of the search array and background scenes dampened the relative saliency of the singleton color distractor. While we are hesitant to conclude that irrelevant context information never influences search performance, given the power of the present experiment, these effects were clearly not present here.

\section{General discussion}

The purpose of the present study was to evaluate whether irrelevant background information impacted visual search. It was proposed by Cosman and Vecera (2013) that search strategies were automatically adopted by participants based on background scenes, and these backgrounds were able to override the bottom-up effects produced by the presence of a singleton color distractor in the search array. Across three experiments, we conceptually replicated the experimental procedures of Cosman and Vecera and varied the type of search task in the test phase across experiments. In Experiment 1, participants performed a singleton search task where they had to find a circle target amongst homogeneous diamond distractors. In Experiment 2, participants performed a feature search task where they had to find a circle target amongst a

\footnotetext{
${ }^{6}$ Due to poor accuracy and the randomization of the context and singleton distractor presence conditions, one participant had an empty cell in one of the key conditions and was subsequently removed from the analysis of the fifth block of Experiment 3.
}

mostly heterogeneous set of distractor shapes. It was revealed that when participants performed the singleton search task of Experiment 1, the presence of a singleton color distractor slowed responding relative to when it was absent while in Experiment 2 the singleton color distractor did not influence performance on the feature search task. Most importantly, reinstating the irrelevant singleton and feature search contexts of the training phase had no influence on performance on either search task. In Experiment 3, we conceptually replicated the procedures of Experiment 1 while using a large sample size and integrating the search arrays and the background displays, and found that irrelevant context still did not influence performance. Ultimately, the results here suggest that irrelevant context information did not determine the search strategies adopted by participants, but that the nature of the search task did.

Given the present results, it remains an open question why Cosman and Vecera (2013) observed one pattern of results and we observed another. Conceptually speaking, it is possible that some proportion of the participants in Cosman and Vecera's study were explicitly aware of the association between the background and search task. That is, it may be their participants explicitly recalled the singleton and feature search tasks when those backgrounds were displayed, and by doing so, it influenced how they prepared for the upcoming search task. This is not to say that we think that the small differences in experimental procedure across the present study and Cosman and Vecera's study produced the different pattern of results, but that whenever you replicate an experiment, there are going to be idiosyncratic differences in the participants tested. In other words, it may be that Cosman and Vecera's study had a particularly diligent group of participants that were more aware of the contingencies between the background and search task relative to our participants. Another possibility is that their effects were due to key conditions not being counterbalanced. That is, both experiments of Cosman and Vecera's study had an odd number of participants $(n=17$; $\mathrm{n}=15$ ), and randomized which of three forest and three city scenes would constitute the singleton and feature search contexts. If by chance the particular scenes associated with the feature search task were more salient than those of the singleton search task, it seems possible that this could dampen the effect of a salient distractor in and of itself, producing a spurious pattern of results indicating that the feature search context diminished the effect of the color singleton distractor.

We also speculate whether the number of trials in the training and test phases could play an important role in whether irrelevant background information can evoke particular search strategies. In a particularly notable study, Leber and Egeth (2006a) rapidly presented gray letters to participants and they had to identify the letter that was presented in a color (e.g., red). Prior to performing this task, one group of participants performed a similar task where they had to identify a particular colored letter (e.g., 
red) amongst heterogeneous colored (e.g., gray, blue, purple, and green) letters (the feature training task) and another group of participants had to identify a target that could be one of five different colors (i.e., red, orange, blue, purple, or green) amongst homogeneously colored grey letters (the singleton training task). Importantly, Leber and Egeth also varied the number of trials of the training task and the nature of the distractor displays that could surround these letters. Leber and Egeth observed that participants that trained the singleton task were less able to accurately identify the target letter if any singleton color was presented in the distractor display. In contrast, the participants that trained the feature task were only impaired by the distractor display if it contained the color of the target letter. In other words, the type of training influenced the nature of distractor interference. Importantly, this pattern of results critically depended on the number of training trials participants performed. When participants performed 320 training trials, this pattern of results was robust, however, when participants performed 40 training trials, this pattern of results was absent. Accordingly, it may be that irrelevant background information has a similar influence - that is, it depends on a high number of training trials. Further, while the block analyses of the present study failed to reveal any effect of the irrelevant background scenes, it is possible that the associations between background and search task are fragile. In other words, it is possible that irrelevant background information evoked an associated search strategy when reinstated, but these associations were quickly dismantled as these backgrounds became paired with a new search task.

It is, however, worth noting that there is one experiment reported in the literature that observed a similar pattern of results as Cosman and Vecera (2013) upon conceptual replication. In a single experiment study, Anderson (2015) used a similar background scene manipulation to evaluate whether these scenes would affect reward learning like in previous studies (Anderson \& Yantis, 2013; Anderson, Laurent, \& Yantis, 2011). Participants performed a training phase where they had to find a particular colored circle amongst heterogeneously colored distractor circles when background scenes (i.e., city and forest) were varied across blocks of trials. For one of the backgrounds scenes, participants were provided with a small monetary reward for correct responses to red targets and were provided no reward for correct responses to green targets. For the other background scene, this reward mapping was reversed. Participants then performed a test phase where they had to find a diamond amongst circles when the background scenes of the training phase were reinstated on a trial-by-trial basis. Importantly, the color targets for the training phase could now appear as one of the distractors. This study showed that search performance was slowed when the high reward distractors were paired with their corresponding background scene, but that performance was unimpaired when distractors were paired with the alternative background scene.
In an interesting turn of events, recent work of Anderson has contradicted the notion that irrelevant context information can impact visual search. Britton and Anderson (2020) used the same basic experimental procedure as Anderson (2015), except that background scenes were now informative of the location of a singleton color distractor across the experimental session. When one background was displayed, a color singleton distractor was presented most of the time in one location, and when the other background was displayed, a color singleton distractor was presented most of the time in an alternative location. Instead of observing that the background scenes modulated the suppression of these high probability distractor locations, it was revealed that the suppression of these high probability distractor locations generalized across backgrounds. That is, participants learned to suppress these high probability distractor locations generally, instead of employing distractor suppression based on the background scene. In keeping with the findings of the present study, Britton and Anderson's work suggests that background information did not influence search task performance, but information relevant to performing the search task (i.e., the location of a salient distractor) did.

Given the findings of the present study, we suspect that the influence of irrelevant context information is tenuous. However, if we are to presume for the moment that it is indeed the case that irrelevant background information influences tasks, we must ask ourselves: why would an adaptive system do this? In order to effectively understand the world around us, it cannot be the case that our understanding is purely the product of simple associations - there must be a system in place that treats some types of information as more important than others. Indeed, this is likely why task-based context effects are generally robust (i.e., contextual cueing, distractor suppression, etc.), and these background-based context effects can be tenuous even when informative of the nature of the task. That is, we must be weighting task relevant information more heavily - whether it be that these context effects are highly depended on attention, which is inevitably occupied by a task when performing it (see Logan, 1988), or there is a system that is organizing and weighting information more heavily if it is relevant to task goals (see Tsotsos, 1990). Ultimately, given the limited processing capacity of the human brain, we suspect that the visual system selects a few relevant features in a scene to conduct more detailed analyses on, which is why background scene information may not always influence performance and task related information impacts us so profoundly.

Acknowledgements Financial support for this study was provided in part by a Natural Science and Engineering Research Council of Canada Discovery Grant 2016-06359 awarded to Jay Pratt. The funding agreement ensured the authors' independence in designing the study, interpreting the data, writing, and publishing the report. The authors report no conflict of interest. 


\section{References}

Allon, A. S., \& Leber, A. B. (2019, November). Experience-driven suppression of irrelevant distractor locations is context dependent. Paper presented at $60^{\text {th }}$ Annual Meeting of the Psychonomic Society. Montreal, Quebec, Canada.

Anderson, B. A. (2015). Value-driven attentional priority is context specific. Psychonomic Bulletin \& Review, 22(3), 750-756.

Anderson, B. A., \& Yantis, S. (2013). Persistence of value-driven attentional capture. Journal of Experimental Psychology: Human Perception and Performance, 39(1), 6-9.

Anderson, B. A., Laurent, P. A., \& Yantis, S. (2011). Value-driven attentional capture. Proceedings of the National Academy of Sciences of the United States of America, 108 (25), 10367-10371.

Bacon, W. F., \& Egeth, H. E. (1994). Overriding stimulus-driven attentional capture. Perception \& Psychophysics, 55(5), 485-496.

Britton, M. K., \& Anderson, B. A. (2020). Specific and persistence of statistical learning in distractor suppression. Journal of Experimental Psychology: Human Perception and Performance, 46(3), 324-334

Brooks, D. I., Rasmussen, I. P., \& Hollingworth, A. (2010). The nesting of search contexts within natural scenes: Evidence from contextual cuing. Journal of Experimental Psychology: Human Perception and Performance, 36(6), 1406-1418.

Chun, M. M., \& Jiang, Y. (1998). Contextual cueing: Implicit learning and memory of visual context guides spatial attention. Cognitive Psychology, 36(1), 28-71.

Cosman, J. D., \& Vecera, S. P. (2013). Context-dependent control over attentional capture. Journal of Experimental Psychology: Human Perception and Performance, 39(3), 836-848.

Cousineau, D. (2005). Confidence intervals in within-subject design: a simpler solution to Loftus and Masson's method. Tutorials in Quantitative Methods for Psychology, 1(1), 42-45.

Crump, M. J. C., Gong, Z., \& Milliken, B. (2006). The context-specific proportion congruent Stroop effect: Location as a contextual cue. Psychonomic Bulletin \& Review, 13(2), 316-321.

Gaspelin, N., \& Luck, S. J. (2018). The role of inhibition in avoiding distraction by salient stimuli. Trends in Cognitive Science, 22(1), 7992.
Jacoby, L. L., Lindsay, S. D., \& Hessel, S. (2003). Item-specific control of automatic processes: Stroop process dissociations. Psychonomic Bulletin \& Review, 10(3), 638-644.

Leber, A. B., \& Egeth, H. E. (2006a). Attention on autopilot: Past experience and attentional set. Visual Cognition, 14(4-8), 565-583.

Leber, A. B., \& Egeth, H. E. (2006b). It's under control: Top-down search strategies can override attentional capture. Psychonomic Bulletin \& Review, 13(1),132-138.

Leber, A. B., Kawahara, J., \& Gabari, Y. (2009). Long-term, abstract learning of attentional set. Journal of Experimental Psychology: Human Perception and Performance, 35(5), 1385-1397.

Leber, A. B., Gwinn, R. E., Hong, Y., \& O’Toole, R. J. (2016). Implicit learned suppression of irrelevant spatial locations. Psychonomic Bulletin \& Review, 23(6), 1873-1881.

Logan, G. D. (1988). Toward an instance theory of automatization. Psychological Review, 95(4), 492-527.

Morey, R. D. (2008). Confidence intervals from normalized data: a correction to Cousineau (2005). Tutorials in Quantitative Methods for Psychology, 4(2), 61-64.

Pashler, H. (1988). Cross-dimensional interaction and texture segregation. Perception \& Psychophysics, 43(4), 307-318.

Theeuwes, J. (1992). Perceptual selectivity for color and form. Perception \& Psychophysics, 51(6), 599-606.

Tsotsos, J. K. (1990). Analyzing vision at the complexity level. Behavioral and Brain Sciences, 13(3), 423-469.

Wang, B., \& Theeuwes, J. (2018). Statistical regularities modulate attentional capture. Journal of Experimental Psychology: Human Perception and Performance, 44(1), 13-17.

Open Practices Statement

The experiments reported in this article were not preregistered. The data for all experiments are publicly available at the Center of Open Science website (osf.io/a37mu). Requests for materials can be sent via email to the corresponding author at brett.cochrane@utoronto.ca.

Publisher's note Springer Nature remains neutral with regard to jurisdictional claims in published maps and institutional affiliations. 